\title{
ABSOLUTE PROPER MOTIONS OF THE DWARF SPHEROIDAL GALAXIES IN DRACO AND URSA MINOR
}

\author{
R.-D. SCHOLZ ${ }^{1}$ and M.J. IRWIN ${ }^{2}$ \\ ${ }^{1}$ WIP-Projekt Astrometrie bei der Universität Potsdam, Germany \\ ${ }^{2}$ Royal Greenwich Observatory, Cambridge, U.K.
}

\begin{abstract}
Palomar and Tautenburg Schmidt plates with a base line of about 35 years have been measured with the Automated Photographic Measuring (APM) system in Cambridge (UK) in order to obtain the proper motions of the Galactic dwarf spheroidal satellites (dSph) in Draco and Ursa Minor with respect to a well defined extragalactic reference frame. The investigations were encouraged by the accuracy level achieved for the mean absolute proper motions of galactic globular clusters $(0.05$ arcsec/century from 25 years base line Tautenburg plate pairs) which is comparable to the expected proper motion of the Draco and Ursa Minor dSph assuming tangential motions of about $100 \mathrm{~km} / \mathrm{s}$. Different methods for the removal of systematic errors in the absolute proper motion introduced by the measuring and reduction process are discussed. The more accurate relative proper motions of individual stars in both dSphs obtained by Stetson (1980) and by Cudworth, Olszewski \& Schommer (1986) provide an external comparison and are also used to obtain the mean absolute proper motion of the dSphs.
\end{abstract}

\section{Introduction}

The kinematics of the galactic globular clusters and of local galaxies are of great interest in the discussion of the dark matter and of the dynamics of the Milky Way. Our present knowledge is mainly based on radial velocity studies. The determination of proper motions with respect to an extragalactic reference frame is needed.

For the nearby dwarf spheroidal galaxies (dSph) in Draco and Ursa Minor accurate relative proper motions have already been determined by Stetson (1980) and by Cudworth, Olszewski \& Schommer (1986). In the latter paper the authors encourage spectral observations of blue objects in order to find some QSOs for the definition of an absolute zero proper motion of the Ursa Minor dSph. We propose an alternative way to obtain the absolute proper motions of these dSphs by measuring large numbers of stars and background galaxies on Palomar and Tautenburg Schmidt plates.

From Tautenburg Schmidt plates with 25 years baseline we have already obtained mean absolute proper motions of the galactic globular clusters $M 3$ and $M 92$ with an accuracy of 0.5 mas/year and used the results for the determination of galactic orbits (Scholz, Odenkirchen \& Irwin 1993, 1994). Nevertheless, the dSphs pose a more challenging problem since with that accuracy the velocity uncertainty is still $\sim 100 \mathrm{~km} / \mathrm{s}$ at the $\sim 70 \mathrm{kpc}$ distance of the dSphs. 


\section{Observations and Measurements}

All plates listed in Table 1 were scanned with the APM. On the first epoch Palomar Sky Survey glass copies the dSphs are situated near the plate corners, whereas on the second epoch Palomar plates also included in this project the dSphs are close to the plate centre. Third epoch plates centred on the dSphs were obtained with the Tautenburg Schmidt telescope in 1991. The plates were taken in different passbands, the Tautenburg plates taken without filter have emulsion (ZU-21) comparable to B plates.

Table 1. Plate material

\begin{tabular}{cccc}
\hline Field & $\begin{array}{c}\text { Plate } \\
\text { Number }\end{array}$ & Epoch & Passband \\
& E1148 & 1954.6 & R \\
\hline \hline Draco & O1148 & 1954.6 & B + U \\
& PS29671 & 1982.5 & Bv \\
& PS29667 & 1982.5 & R \\
& T7564 & 1991.5 & B \\
& T7664 & 1991.7 & no filter (B) \\
& T7717 & 1991.8 & V \\
& E1575 & 1956.2 & R \\
UMi & O1575 & 1956.2 & B + U \\
& PS29610 & 1982.4 & Bv \\
& PS29666 & 1982.5 & R \\
& T7585 & 1991.6 & B \\
& T7651 & 1991.7 & no filter (B) \\
& T7693 & 1991.8 & V \\
\hline
\end{tabular}

The measured objects were classified into stars, nonstellar objects, noise images and merged objects using standard APM software. The internal magnitude calibration as described by Bunclark \& Irwin (1983) was applied to the measurements. An external calibration was made fitting the APM magnitudes on the Tautenburg B plates to the B magnitudes given in Baade \& Swope (1961) and in Cudworth, Olszewski \& Schommer (1986).

In both dSph about 500 objects were investigated by Stetson (1980) and by Cudworth, Olszewski \& Schommer (1986). These objects cover only the central part of the dSph (within $11 \times 11$ arcmin in Draco and $17 \times 17$ arcmin in Ursa Minor). On our deepest plates we identified 361 of these objects from the visual inspection of the Draco finding charts of Baade \& Swope (1961) and 444 objects in Ursa Minor from matching our coordinates with those given by Cudworth, Olszewski \& Schommer (1986).

The plate limit of our deepest plates was $B \sim 21.7$ (1st epoch R-plate, 103a-E emulsion) and $B \sim 21.3$ (Bv-plate, IIIa-J emulsion) respectively for Draco and UMi. Whereas in the Draco field all Palomar plates reached fainter magnitudes than the Tautenburg ones this was not the case in the UMi field. The Tautenburg V plates did not go deep enough so that they were excluded from the proper motion determination. Unfortunately, the limiting magnitude on the first epoch $R$ plate in the Ursa Minor field was also a few 1/10 magnitudes lower.

Only nonsaturated, faint stars and galaxies were selected in order to avoid magnitude 
dependent systematic errors in the proper motions. The saturation of stellar images begins some 3 to 4 magnitudes above the plate limit so that only a few stars in the dSphs were saturated. A further restriction of the magnitude intervals of the galaxies was used to ensure more reliable classifications.

For the differential proper motion determination we combined the plates of similar colour in pairs. Due to the inhomogeneous plate material we did not try to include all plates in a central overlap method. The maximum symmetric overlap field with the dSph in the centre was about 3 (Draco) and 4 (UMi) square degrees except for the Bv-(B) pair in the Ursa Minor field where about 7 square degrees was available.

\section{Determination of Absolute Proper Motions}

The plate-to-plate solution for each pair of plates was obtained with all faint stars outside the dSph region using 3rd order polynomials and the method of stepwise regression described in Hirte et al. (1990). The same stars were used in the iterative procedure of removing the errors of the measuring machine (cf. Evans 1988) and large field systematic effects including both residual model errors and possible proper motion variations over the field.

We preferred to model the more complicated differential geometry from Palomar to Tautenburg Schmidt plates by faint stars instead of the galaxies in order not only to yield a higher accuracy in the global solution but also in the systematic error removal. The proper motions of the stars in the dSph region and in the outer field were then converted to absolute proper motions by subtracting the mean (median) 'proper motion' of the galaxies. The $\mathrm{dSph}$ regions were defined as $26 \times 26$ arcmin in Draco and $34 \times 26$ arcmin in Ursa Minor.

The mean (median) of the absolute proper motions of the dSph was corrected for the contamination with field stars in the dSph region. The number of field stars $n_{f}$ in the dSph region was estimated from the stellar density in the outer field.

$$
\mu_{a b s}^{d S p h}=\left(1+\frac{n_{f}}{n_{d S p h}}\right) \mu_{r e l}^{d S p h+f i e l d}+\frac{n_{f}}{n_{d S p h}} \mu_{r e l}^{\text {field }}-\mu_{r e l}^{\text {gal }}
$$

According to the different epoch differences and object numbers, there are different rms errors in the mean (median) of the proper motions of $\mathrm{dSph}$ and field stars but especially for the galaxies. Therefore, rather different weights were given to the plate pairs (Table 2).

Considering the non-gaussian proper motion distribution function, the median was a better choice compared to the mean which depends more severely on outliers.

\section{Results}

The weighted means from three pairs of plates together with their formal errors are listed in Table 3. The first row shows the results obtained in the way described above, i.e. the mean absolute proper motion in the $\mathrm{dSph}$ region corrected for the contamination by field stars. The second row contains a second solution obtained with alternative, smaller samples of stars and galaxies within a further reduced magnitude interval. The statistically obtained numbers of dSph stars varied for 
the different pairs of plates as a function of their limiting magnitudes, from 250 to 500 in the first solution (with about 100 stars less in the second solution). There were also about 100 less galaxies in the second solution compared with the first solution (see Table 2).

Table 2. Different weights of the plate pairs (1st solution)

\begin{tabular}{cccccc}
\hline Field & $\begin{array}{c}\text { plate } \\
\text { pair }\end{array}$ & $\begin{array}{c}\text { base } \\
\text { line } \\
\text { [years] }\end{array}$ & $\begin{array}{c}\text { No of } \\
\text { galaxies }\end{array}$ & $\begin{array}{c}\text { Error of median } \\
\text { absolute dSph proper motion } \\
\text { in } \boldsymbol{x} \\
\text { in } \boldsymbol{y} \\
\text { [mas/year] }\end{array}$ & $\begin{array}{c}\text { Weight } \\
\text { of } \\
\text { the pair }\end{array}$ \\
\hline \hline Draco & R-R & 27.9 & 819 & 0.53 & \\
& & & & 0.59 & 1.55 \\
& Bv-(B) & 9.2 & 420 & 2.19 & 1.36 \\
& & & & 2.28 & 0.09 \\
& B+U-B & 37.0 & 455 & 0.54 & 0.09 \\
UMi & & & & 0.52 & 1.36 \\
& R-R & 26.3 & 796 & 0.74 & 1.55 \\
& & & & 0.76 & 1.13 \\
& Bv-(B) & 9.3 & 1280 & 1.34 & 0.33 \\
& & & & 1.09 & 0.47 \\
& B+U-B & 35.4 & 574 & 0.62 & 1.54 \\
& & & 0.61 & 1.52 \\
\hline
\end{tabular}

The next two rows in Table 3 are the results of the correction of the relative proper motions of Stetson (1980) and Cudworth, Olszewski \& Schommer (1986) on the basis of two different samples of reference galaxies. The number of common stars (in the central region of the dSphs only) varied for the different plate pairs from 80 to 140 in Draco and from 110 to 180 in Ursa Minor. All stars independent of their membership probabilities were included in the determination of the mean difference (our absolute p.m. minus the relative p.m.). The absolute proper motion of the dSph was then determined as the sum of the proper motion difference of common stars plus the mean relative proper motion of high probable dSph members in the catalogues of Stetson (1980) and Cudworth, Olszewski \& Schommer (1986). The total value of the mean relative proper motion of the $\mathrm{dSph}$ in these catalogues was small (0.15 to 0.25 mas/year).

The last row in Table 3 represents a realistic estimate of the errors in the mean absolute proper motion of the dSphs. Despite the large errors, at least the direction of the absolute proper motion of the dSphs seems to be determined. After correcting for the reflex solar motion about the centre of our Galaxy (i.e. $220 \mathrm{~km} / \mathrm{s}$ in the direction $1=90, b=0$ ) the galactocentric proper motions of the dSphs are $0.9 \pm 0.5,1.0 \pm 0.5$ for Draco and $1.0 \pm 0.8,0.8 \pm 0.8$ for Ursa Minor. These are consistent within the errors with a polar orbit along the direction of the Magellanic Stream in the same orbital direction as the recent determination of the motion of the LMC (Lin - personal communication). 
Table 3. Weighted mean absolute proper motion of the dSphs from three pairs of plates [mas/year]

\begin{tabular}{lcccc}
\hline & \multicolumn{2}{c}{ Draco } & \multicolumn{2}{c}{ UMi } \\
& $\mu_{x}$ & $\mu_{y}$ & $\mu_{x}$ & $\mu_{y}$ \\
\hline \hline $\begin{array}{l}\text { 1st solution } \\
\text { 2nd solution (smaller mag } \\
\text { interval) }\end{array}$ & $0.70 \pm 0.77$ & $1.01 \pm 0.14$ & $-0.14 \pm 1.28$ & $1.33 \pm 0.55$ \\
$\begin{array}{c}\text { 1st solution compared with } \\
\text { rel. p.m. }\end{array}$ & $1.02 \pm 0.24$ & $1.34 \pm 0.39$ & $1.26 \pm 2.33$ & $1.15 \pm 0.50$ \\
$\begin{array}{c}\text { 2nd solution compared with } \\
\text { rel. p.m. }\end{array}$ & $0.59 \pm 0.62$ & $1.22 \pm 0.34$ & $0.58 \pm 1.05$ & $1.20 \pm 0.54$ \\
average & $0.16 \pm 0.31$ & $0.95 \pm 0.26$ & $0.31 \pm 1.02$ & $0.95 \pm 0.24$ \\
\hline
\end{tabular}

\section{References}

Baade, W. and Swope, H.H., 1961. Astron. J., 66, 300.

Bunclark, P.S. and Irwin, M.J., 1983. In 'Statistical Methods in Astronomy', Proc. of an International Colloquium, Strasbourg 13-16 Sept. 1993 (ESA SP-201), ed. E.J. Rolfe, p. 195.

Cudworth, K.M., Olszewski, E.W. and Schommer, R.A., 1986. Astron. J., 92, 766.

Evans, D.W., 1988. PhD Thesis, Cambridge.

Hirte, S., Dick, W.R., Schilbach, E and Scholz, R.-D., 1990. In 'Errors, Uncertainties and Bias in Astronomy', eds. C. Jaschek and F. Murtagh, Cambridge University Press, p. 343.

Scholz, R.-D., Odenkirchen, M. and Irwin, M.J., 1993. Mon. Not. R. astron. Soc., in press.

Scholz, R.-D., Odenkirchen, M. and Irwin, M.J., 1994. Mon. Not. R. astron. Soc., submitted.

Stetson, P.B., 1980. Astron. J., 85, 387. 